Bryn Mawr College

Scholarship, Research, and Creative Work at Bryn Mawr

College

Psychology Faculty Research and Scholarship

Psychology

2011

\title{
Adolescents Prefer More Immediate Rewards When in the Presence of their Peers
}

Lia O'Brien

Dustin Albert

Bryn Mawr College, walbert@brynmawr.edu

Jason Chein

Laurence Steinberg

Let us know how access to this document benefits you.

Follow this and additional works at: http://repository.brynmawr.edu/psych_pubs

Part of the Psychology Commons

\section{Custom Citation}

O'Brien, L., Albert, D., Chein, J. and Steinberg, L. (2011), Adolescents Prefer More Immediate Rewards When in the Presence of their Peers. Journal of Research on Adolescence, 21: 747-753.

This paper is posted at Scholarship, Research, and Creative Work at Bryn Mawr College. http://repository.brynmawr.edu/psych_pubs/55

For more information, please contact repository@brynmawr.edu. 
Adolescents Prefer More Immediate Rewards When In The Presence Of Their Peers

Lia O’Brien, Dustin Albert, Jason Chein, and Laurence Steinberg

Temple University 


\begin{abstract}
Adolescents take more risks in the presence of their peers, but the mechanism through which peer presence affects risky decision making is unknown. We propose that the presence of peers increases the salience of the immediate rewards of a risky choice. The current study examined the effect of peer presence on reward sensitivity in a sample of 100 late adolescents ages 18 through $20(M=18.5)$ using a delay discounting task, which assesses an individual's preference for immediate versus delayed rewards. Participants were randomly assigned to complete the task alone or with two same-age, same-sex peers observing. Consistent with our prediction, adolescents demonstrated a greater preference for immediate rewards when with their peers than when alone. Heightened risk taking by adolescents in the company of their friends may be due in part to the effect that being with one's peers has on reward sensitivity.
\end{abstract}


Adolescents Prefer More Immediate Rewards When In The Presence Of Their Peers

It is widely agreed among experts in the study of adolescent health and development that the greatest threats to the well-being of young people in industrialized societies come from preventable and often self-inflicted causes, including automobile and other accidents (which together account for nearly half of all fatalities among American youth), violence, drug and alcohol use, and sexual risk-taking (Blum \& Nelson-Mmari, 2004; Williams, Holmbeck, \& Greenley, 2002). It is also the case that adolescents and young adults engage in more risky behavior than older adults: Adolescents and young adults are more likely than adults over 25 to binge drink, smoke cigarettes, have casual sex partners, engage in violent and other criminal behavior, and have fatal or serious automobile crashes, the majority of which are caused by risky driving or driving under the influence of alcohol (Steinberg, 2008).

One of the hallmarks of risk-taking in adolescence is that it is far more likely than that of adults to occur in groups. This is especially the case with respect to substance use and abuse, which during adolescence occurs primarily in the company of peers (Chassin, Hussong, \& Beltran, 2009). In addition, research on automobile accidents indicates that the presence of same-aged passengers in a car driven by an adolescent driver significantly increases the risk of a serious accident (Simons-Morton, Lerner, \& Springer, 2005). And statistics compiled by the Federal Bureau of Investigation show that youths are far more likely than adults to commit crimes in groups than by themselves (Zimring, 1998).

Although one possible explanation for the fact that relatively more adolescent risk-taking takes place with peers is the fact that during this period individuals simply spend more time with friends than adults do, recent experimental evidence suggests that the mere presence of peers 
increases risk-taking among adolescents and college-aged youths, but not adults. Gardner and Steinberg (2005) examined risk-taking among adolescents, undergraduates, and adults who were randomly assigned to engage in a video driving game alone or in the presence of two friends. They found that adolescents and undergraduates, but not adults, took more risks and rated the benefits of various risky behaviors (e.g., having unprotected sex) relatively higher when in the presence of their peers than when alone. Indeed, the presence of peers doubled the number of risky behaviors adolescents engaged in, and increased risk-taking among the undergraduates by 50 percent.

Many current theories of risky decision-making among young people derive from behavioral economics and frame the process as one in which individuals perceive, assess, and weigh the relative costs and benefits of various choices of action (Gruber, 2001). The specific mechanism by which this process may be affected by the presence of peers is unknown, however. Based on several findings in the extant literature, we propose that one possible pathway by which the presence of peers may bias adolescents and youths toward risk-taking is by increasing the salience of the potential immediate reward of a risky decision.

Evidence from a variety of behavioral and neuroimaging paradigms is consistent with this proposition. For instance, as noted above, Gardner \& Steinberg (2005) found that adolescents not only took more risks when peers were present, but focused more on the benefits than the costs of risky decisions under this condition. Furthermore, a recent study by Cauffman and colleagues (Cauffman, Shulman, Steinberg, Claus, Banich, et al., 2010), using a variant of the Iowa Gambling Task, suggests that adolescents show greater behavioral sensitivity to reward than to punishment feedback. Specifically, participants were given the choice to "play or pass" from each of four decks of cards, with each "play" decision resulting in a monetary gain or loss; 
adolescents, to a greater degree than adults, adjusted their behavior more in response to feedback indicating gains than to feedback indicating losses, consistent with the adolescents' relatively greater sensitivity to rewards than punishments. Indeed, recent fMRI studies examining neural activity during performance on a delayed reward task have found that adolescents actually may be hypersensitive to anticipated rewards in comparison to children or adults (e.g., Galvan, Hare, Parra, Penn, Voss, et al., 2006). Finally, suggestive findings from our lab (Chein, Albert, O'Brien, Uckert, \& Steinberg, under review) indicate that the presence of peers specifically sensitizes activation of the brain's incentive processing system (e.g., ventral striatum and orbitofrontal cortex) as adolescents render decisions about risk-taking (i.e., whether to cross an intersection when a traffic signal was yellow in a driving game), but has no such impact on adults.

The present study uses a delay discounting task, which examines preference for immediate versus delayed rewards, to test the hypothesis that the presence of peers increases adolescents' preference for immediate rewards (see Figure 1). In this paradigm, the participant is presented with a series of choices between a relatively small, immediate reward (e.g., \$200 today) and a larger, delayed reward (e.g., \$1,000 in 1 year). To the degree that respondents prefer smaller, immediate rewards, they are said to "discount" the value of the larger reward due to the delay in reward delivery. An extensive empirical literature links the propensity to discount delayed rewards to behaviors and traits indicative of preference for immediate rewards, such as substance use (e.g., Petry, 2002) and weaker future orientation (e.g., Steinberg, Graham, O’Brien, Woolard, Cauffman, et al., 2009).

Notably, however, these previous studies assess delay discounting among individuals only when they are alone. Given the fact that adolescent risk-taking tends to occur in the 
company of peers, and in light of the Gardner and Steinberg (2005) experiment described earlier, in the present study, we randomly assigned late adolescent participants to perform the discounting task either alone or with their friends watching them. To our knowledge, this is the first study to examine social influences on delay discounting. Our hypothesis is that the presence of peers will lead individuals to evince a stronger preference for immediate over delayed rewards.

Method

\section{Participants}

The sample included 100 participants (52 females), ages 18 through $20(M=18.47) ; 57 \%$ White, 21\% Black, 19\% Asian, 2\% Hispanic, and 1\% Other. All participants were recruited from undergraduate introductory psychology courses and fliers posted on the campus of a large urban university. Recruited individuals were asked to bring two same-sex friends when reporting for the study. These groups of three were then randomly assigned to a peer condition or to an alone condition. In the peer condition, one member of the group was randomly chosen as the primary participant and was seated directly in front of a desktop computer to complete the experiment while the two peers observed, resulting in data from one participant per group $(n=45)$. The peers were seated in adjacent seats on either side of the participant, and were allowed to communicate with the participant as the tasks were performed. In the alone condition, the three participants each completed the battery of measures alone in three separate rooms, resulting in data from three individuals per group. After collecting 19 groups of 3 individuals who had been randomly assigned to take the test battery alone, data for 2 participants were discarded due to file corruption, resulting in a final sample in the alone condition of $n=55$. The two experimental 
groups did not differ significantly with respect to age $(t(98)=0.99, p=.32)$, gender $\left(\chi^{2}(1)=\right.$ $0.06, p=.81)$, maternal education $(t(98)=1.28, p=.21)$, or ethnicity $\left(\chi^{2}(4)=4.65, p=.33\right)$.

Participants completed a battery of computerized tasks, which included a delay

discounting task, as well as a set of questionnaires, and were administered an IQ test. The entire battery took approximately two hours to complete. Participants were told that they would receive $\$ 25$ or research credit for participation in the study and that they could receive an additional $\$ 5$ based on their performance on the computerized tasks, an incentive included to maximize attention and motivation throughout the relatively lengthy experiment. Although other tasks in the battery involved explicit performance goals (e.g., fast completion, minimal errors), the delay discounting task was clearly introduced as a measure of preference, not performance, and it was clear that the choices were hypothetical, not real (see below); therefore, we have no reason to believe that the promised incentive influenced preference judgments. In reality, all participants received the $\$ 5$ bonus at the end of the experiment.

\section{Measures}

Delay Discounting. The delay discounting task was administered on a laptop computer. In our adaptation of the task, the amount of the delayed reward was held constant at $\$ 1,000$. We varied the length of the delay in 6 blocks ( 1 day, 1 week, 1 month, 3 months, 6 months, and 1 year), presented in a random order (see Figure 1). For each block, the starting value of the immediate reward was $\$ 200, \$ 500$, or $\$ 800$, randomly determined for each participant. The respondent was asked to choose between an immediate reward of a given amount and a delayed reward of $\$ 1,000$ (e.g., "Would you rather have $\$ 200$ today or $\$ 1,000$ in six months?”). If the immediate reward was preferred, the subsequent question presented an immediate reward midway between the prior one and zero (i.e., a lower amount). If the delayed reward was 
preferred, the subsequent question presented an immediate reward midway between the prior one and $\$ 1,000$ (i.e., a higher amount). Participants then worked their way through these ascending and descending choices, choosing between the reward just rejected and the previously rejected lower or higher reward, until their responses converged and their preference for the immediate and delayed reward were equal, at a value reflecting the "discounted" value of the delayed reward (i.e., the subjective value of the delayed reward if it were offered immediately; Green, Myerson, \& Macaux, 2005), referred to as the indifference point (Ohmura, Takahashi, Kitamura, $\&$ Wehr, 2006). For each individual, we computed the indifference point for each delay interval, the average indifference point, and the discount rate. The discount rate $(k)$ is an index of the degree to which an individual devalues a reward as a function of the length of delay to receipt, which we computed using the standard equation, $\mathrm{V}=\mathrm{A} /(1+k \mathrm{D})$, where $\mathrm{V}$ is the subjective value of the delayed reward (i.e., the indifference point), A is the actual amount of the delayed reward, $\mathrm{D}$ is the delay interval, and $k$ is the discount rate. Because, as is usually the case, the distribution of $k$ was highly positively skewed (5.95), we employed a natural log transformation to reduce skewness to an acceptable level (-1.08). An individual with a relatively lower indifference point and/or a relatively higher (steeper) discount rate is relatively more oriented toward the immediate than the future. In the present sample, the correlation between the average indifference point and discount rate is $r=-.56, p<.01$.

Intelligence. Performance on delay discounting tasks has been found to be correlated with IQ, with less intelligent individuals demonstrating a stronger preference for immediate rewards. The Wechsler Abbreviated Scale of Intelligence (WASI) (Psychological Corporation, 1999) was used to produce an estimate of general intellectual ability based on two (Vocabulary and Matrix Reasoning) of the four subtests. The Vocabulary subtest requires the participant to 
define a variety of words and used to generate a measure of crystallized ability. The Matrix Reasoning subtest provides a measure of nonverbal fluid abilities. The two-subtest form of the WASI was used because it can be administered in approximately 15 minutes and is correlated with both the Wechsler Intelligence Scale for Children $(r=.81)$ and the Wechsler Adult Intelligence Scale $(r=.87)$. As in other studies, IQ was significantly correlated with average indifference point $(r=.28, p<.01)$ and discount rate $(\mathrm{r}=-.23, p<.05)$. The average IQ of the participants in each experimental group did not differ $($ alone $=107.92$, peer $=103.51 ; F(1,93)=$ 3.56, ns).

Because there were no differences between the experimental conditions with respect to age, maternal education, gender, race, or IQ, it was not necessary to control for these factors in analyses of experimental effects; however, we did test for interactions between these variables and experimental condition.

\section{Results}

Indifference Point. As noted earlier, we computed indifference points based on participants' choices between immediate and delayed rewards for offers at 6 different delay intervals (i.e., 1 day, 1 week, 1 month, 3 months, 6 months, and 1 year), such that each indifference point represents the average value for which an immediate reward was subjectively equivalent to a $\$ 1000$ reward delivered after the corresponding delay. To test the hypothesis that individuals making decisions in the presence of their peers would evince a greater preference for immediate rewards than individuals making decisions alone, we conducted a repeated measures Analysis of Variance (ANOVA) on individuals' indifference points, with experimental condition as the between-subjects factor and the six delay intervals as the within-subjects factor. As in virtually all studies of delay discounting, we found a main effect of the delay interval factor 
$(F(5,490)=160.56, p<.01)$, indicating that individuals' indifference points decrease as the delay interval increases. Furthermore, consistent with our hypothesis, we found a significant main effect for condition $(F(1,98)=4.02, p<.05, d=.40)$, such that participants who performed the task with their friends watching evinced a significantly lower average indifference point $(\$ 603.84)$ than did those who performed the task alone (\$675.05) (see Figure 2). Although betweensubjects effects were not moderated by age, maternal education, gender, ethnicity, or IQ, a marginally significant interaction between experimental condition and the repeated time factor $(F(5,490)=1.91, p=.09)$ suggests that the degree to which reward preference differs between conditions is moderated by the length of the delay interval. Results from follow-up univariate ANOVAs at each of the six delay intervals confirm this interpretation, indicating that differences between the peer and alone conditions in indifference points are significant only at delay intervals of 3 months $(F(1,98)=5.23, p<.05, d=.45)$ and 6 months $(F(1,98)=6.08, p<.05$, $d=.48$ ) (see Figure 3).

Discount Rate. Consistent with the pattern of results reported for indifference points, results from a univariate ANOVA examining condition differences in discount rate $(\mathrm{k})$ demonstrated a significant difference between the discount rates of those who performed the task in the presence of their peers and those who performed it alone $(F(1,92)=6.48, p<.05, d=.50)$. Specifically, participants who were observed by their peers more strongly discounted the value of delayed rewards, indicating a stronger preference for more immediate rewards than shown by those who performed the task alone. In other words, the presence of peers causes adolescents to find delayed rewards less valuable than smaller immediate rewards. This difference was not moderated by age, maternal education, gender, ethnicity, or IQ, and was significant with or without IQ controlled. 


\section{Discussion}

In many risky situations, decision makers must weigh the prospect of an immediate reward (getting high, the pleasure of unprotected sex, the thrill of driving fast) against the possibility of a negative consequence in the future (a hangover, a pregnancy, a speeding ticket). The present study provides one clue as to why risk taking is more likely to occur when adolescents are with their friends than when they are alone: the mere presence of peers increases the degree to which adolescents prefer immediate rewards. When evaluating whether to take a risk, this shift in reward processing could plausibly bias attention toward the immediate benefits of a risky choice and away from the long-term benefits (e.g., health, security) of a safe choice, potentially tipping the scales toward risky behavior.

What processes underlie this peer influence on reward preference? Although admittedly speculative, we propose that peer presence may function at the neurobiological level to sensitize adolescents and youths (more so than adults) to the value of immediate rewards. This hypothesis is consistent with findings from a recent experiment with college undergraduates that examined the effects of unconsciously primed emotional states on consumption of a novel, non-alcoholic beverage (Winkielman, Berridge, \& Wilbarger, 2005). Following subliminal presentation of either happy or angry faces, participants who had viewed happy faces chose to pour and drink more of the beverage than those who had viewed angry faces, despite participants reporting no differences in subjective mood. Given that exposure to a wide variety of socio-emotional stimuli, including facial expressions, is known to engage dopaminergic pathways involved in incentive processing and reward valuation (e.g., ventral striatum and orbitofrontal cortex; Adolphs, 2003; Fliessbach, Weber, Trautner, Dohmen, Sunde, et al., 2007), the authors suggest that exposure to positive emotion faces sensitized their participants' neural circuitry to respond 
to the reward value of the beverage, an effect they refer to as approach sensitization. This account offers a useful framework for interpreting the findings from the present study. In the peer condition, participants were exposed to two same-age friends sitting close by on either side, a potentially salient socio-emotional stimulus. It is possible that the presence of friends, like the exposure to positive emotion faces in the Winkielman et al. study, heightened activity in participants' incentive processing circuitry, sensitizing them to the approach value of the immediate rewards offered in the delay discounting task.

It is important to note that this hypothesized effect of peers on approach sensitization does not preclude the possibility that peer presence also (or instead) influences adolescents' choices through other mechanisms. For example, the presence of peers could plausibly increase the salience of the potential implications of adolescents' choices for their social status. When choosing between a risky and a safe decision (as in the Gardner and Steinberg [2005] experiment discussed earlier, in which participants could risk crashing a car in order to gain more points), adolescents may favor the risky choice to appear courageous. However, whereas it is easy to imagine that running a stoplight in a driving game in order to gain a potential reward could impress an adolescent's friends as a bold act, it is not clear that 19-year-olds would be more favorably impressed by the choice of $\$ 500$ now than by the choice of $\$ 1000$ in six months, especially in the context of a study where participants were told that their decisions to choose immediate versus delayed rewards were hypothetical (i.e., the participants were made aware that they would not be receiving the monetary values from which they were choosing). Nonetheless, an important goal for future research is to further explore alternative explanations for the peer effect observed in the present study. 
It is also important to acknowledge that because we did not study other age groups, we cannot say whether the phenomenon observed here is unique to young people. However, several lines of evidence suggest that the hypothesized reward sensitization effect of peers on incentive processing may be particularly strong among adolescents. Neuroimaging studies of social information processing have shown greater activation in response to social stimuli among adolescents than adults in incentive processing regions (Blakemore, 2008; Burnett \& Blakemore, 2009; Guyer, McClure-Tone, Shiffrin, Pine, \& Nelson, 2009), consistent with reports that adolescents rate peer interactions as their most rewarding experiences (Csikszentmihalyi, Larson, \& Prescott, 1977). Indeed, the heightened salience and rewarding nature of peers during adolescence appears highly conserved across species; adolescent rats also spend more time than younger or older rats interacting with peers, while showing evidence that such interactions are highly rewarding (Spear, 2009). In sum, evidence points to adolescence as a time of heightened responsiveness to social rewards. Although the precise neural mechanisms by which such responsiveness might sensitize individuals to the value of other types of rewards is not clear, to the degree that this sensitization effect occurs as a result of exposure to friends, it may have more impact among adolescents than adults. An important goal for future research is to examine qualities of the "audience" (e.g., age, gender, social status) that might moderate the peer presence effect, perhaps even reversing its directionality; for instance, perhaps adolescents would be biased toward choosing delayed rewards if observed by their parents.

In closing, it is instructive to put the magnitude of the peer effect observed in the present study in context. To do so, we have re-presented the findings from the current study side by side with findings from an earlier normative study of age differences in delay discounting, in which all participants performed the same task employed here, but only alone (Steinberg, et al., 2009). 
In the earlier study, we demonstrated that younger adolescents (age 15 and younger) show a greater preference for immediate rewards than do older adolescents and adults. In the present study, when they were alone, participants evinced an average indifference point that is virtually identical to that evinced by the comparable age group in the earlier study (see Figure 4).

However, in the present study, when they were with their friends, the late adolescent participants exhibited a pattern of discounting comparable to the 14 and 15 year-olds in the earlier study. It appears that, at least in this respect, the presence of peers transforms the choices of a late adolescent into ones that are similar to those of a less mature teenager. 


\section{Author Note}

This research was supported by the John D. and Catherine T. MacArthur Foundation

Research Network on Adolescent Development and Juvenile Justice. Address correspondence to Lia O’Brien, Department of Psychology, Temple University, Philadelphia, PA, 19122, or via email, at lia.obrien@temple.edu. 


\section{References}

Adolphs, R. (2003). Cognitive neuroscience of human social behavior. Nature Reviews Neuroscience, 4, 165-178.

Blakemore, S-J. (2008). The social brain in adolescence. Nature Reviews Neuroscience, 9, 267277.

Blum, R., \& Nelson-Mmari, K. (2004). The health of young people in a global context. Journal of Adolescent Health, 35, 402-418.

Burnett, S., \& Blakemore, S-J. (2009). Functional connectivity during a social emotion task in adolescents and adults. European Journal of Neuroscience, 29, 1294-1301.

Cauffman, E., Shulman, E., Steinberg, L., Claus, E., Banich, M., Graham, S., \& Woolard, J. (2010). Age differences in affective decision making as indexed by performance on the Iowa Gambling Task. Developmental Psychology, 46, 193-207.

Chassin, L., Hussong, A., \& Beltran, I. (2009). Adolescent substance use. In R. Lerner \& L. Steinberg (Eds.), Handbook of adolescent psychology ( $3^{\text {rd }}$ ed., Vol. 1), pp. 723-764. New York: Wiley.

Chein, J., Albert, D., O'Brien, L., Uckert, K., \& Steinberg, L. (under review). Hyper-responsivity to anticipated rewards underlies the peer influence on adolescent risk taking.

Csikszentmihalyi, M., Larson, R., \& Prescott, S. (1977). The ecology of adolescent experience. Journal of Youth and Adolescence, 6, 218-294.

Fliessbach, K., Weber, B., Trautner, P., Dohmen, T., Sunde, U., Elger, C., \& Falk, A. (2007). Social comparison affects reward-related brain activity in the human ventral striatum. Science, 318, 1305-1308. 
Galvan, A., Hare, T. A., Parra, C. E., Penn, J., Voss, K. et al. (2006). Earlier development of the accumbens relative to orbitofrontal cortex might underlie risk-taking behavior in adolescents. Journal of Neuroscience, 26(25), 6885-6892.

Gardner, M. \& Steinberg, L. (2005). Peer influence on risk taking, risk preference, and risky decision making in adolescence and adulthood: An experimental study. Developmental Psychology, 41, 625-635.

Green, L., \& Myerson, J. (2004). A discounting framework for choice with delayed and probabilistic rewards. Psychological Bulletin, 130, 769-792.

Green, L., Myerson, J., \& Macaux, E. (2005). Temporal discounting when the choice is between two delayed rewards. Journal of Experimental Psychology, 31, 1121-1133.

Gruber, J. (Ed.). (2001). Risky behavior among youths: An economic analysis. Chicago: University of Chicago Press.

Guyer, A., McClure-Tone, E., Shiffrin, N., Pine, D., \& Nelson, E. (2009). Probing the neural correlates of anticipated peer evaluation in adolescence. Child Development, 80, 10001015.

Ohmura, Y., Takahashi, T., Kitamura, N., \& Wehr, P. (2006). Three-month stability of delay and probability discounting measures. Experimental and Clinical Psychopharmacology, 14, $318-328$.

Petry, N. (2002). Discounting of delayed rewards in substance abusers: relationship to antisocial personality disorder. Psychopharmacology, 162, 1432-2072.

Psychological Corporation (1999). Wechsler Abbreviated Scale of Intelligence. San Antonio, TX: Psychological Corporation. 
Simons-Morton, B., Hartos, J., Leaf, W., \& Preusser, D. (2005). Persistence of effects of the Checkpoints Program on parental restrictions on teen driving privileges. American Journal of Public Health, 95, 447-452.

Spear, L. (2009). The behavioral neuroscience of adolescence. New York: Norton.

Steinberg, L. (2008). A neurobiological perspective on adolescent risk-taking. Developmental Review, 28, 78-106.

Steinberg, L., Graham, S., O’Brien, L., Woolard, J., Cauffman, E., \& Banich, M. (2009). Age differences in future orientation and delay discounting. Child Development, 80, 28-44.'

Williams, P., Holmbeck, G., \& Greenley, R. (2002). Adolescent health psychology, Journal of Consulting and Clinical Psychology, 70, 828-842.

Winkielman, P., Berridge, K. C., \& Wilbarger, J. L. (2005). Unconscious affective reactions to masked happy versus angry faces influence consumption behavior and judgments of value. Personality and Social Psychology Bulletin, 1, 121-135.

Zimring, F. (1998). American youth violence. New York: Oxford University Press. 


\section{Figure Captions}

Figure 1. Example of Flow of Decision Alternatives in Delay Discounting Task

Figure 2. Average Indifference Points for 18- to 20-Year-Olds Alone and With Peers

Figure 3. Discount Functions of 18- to 20-Year-Olds Alone and With Peers

Figure 4. Average Indifference Points as a Function of Age and Peer Presence 
Figure 1. Example of Flow of Decision Alternatives in Delay Discounting Task

QUESTION: Would you rather have $\$ 200$ today or $\$ 1,000$ in six months?

ANSWER: $\quad \$ 1,000$ in 6 months

QUESTION: $\$ 600$ today or $\$ 1,000$ in six months?

ANSWER: $\quad \$ 600$ today

QUESTION: $\$ 400$ today or $\$ 1,000$ in six months?

ANSWER: $\quad \$ 1,000$ in six months

QUESTION: $\$ 500$ today or $\$ 1,000$ in six months?

ANSWER: $\quad \$ 1,000$ in six months

QUESTION: $\$ 550$ today or $\$ 1,000$ in six months?

ANSWER: $\quad \$ 550$ today

QUESTION: $\$ 525$ today or $\$ 1,000$ in six months?

ANSWER: $\quad \$ 1,000$ in six months

QUESTION: $\$ 538$ today or $\$ 1,000$ in six months?

ANSWER: $\quad \$ 538$ today

QUESTION: $\$ 532$ today or $\$ 1,000$ in six months?

ANSWER: $\quad \$ 1,000$ in six months

QUESTION: $\$ 535$ today or $\$ 1,000$ in six months?

ANSWER: $\quad \$ 535$ today

QUESTION: $\$ 534$ today or $\$ 1,000$ in six months?

ANSWER: $\quad \$ 534$ today

INDIFFERENCE POINT REACHED 
Figure 2. Average Indifference Points for 18- to 20-Year-Olds Alone and With Peers.

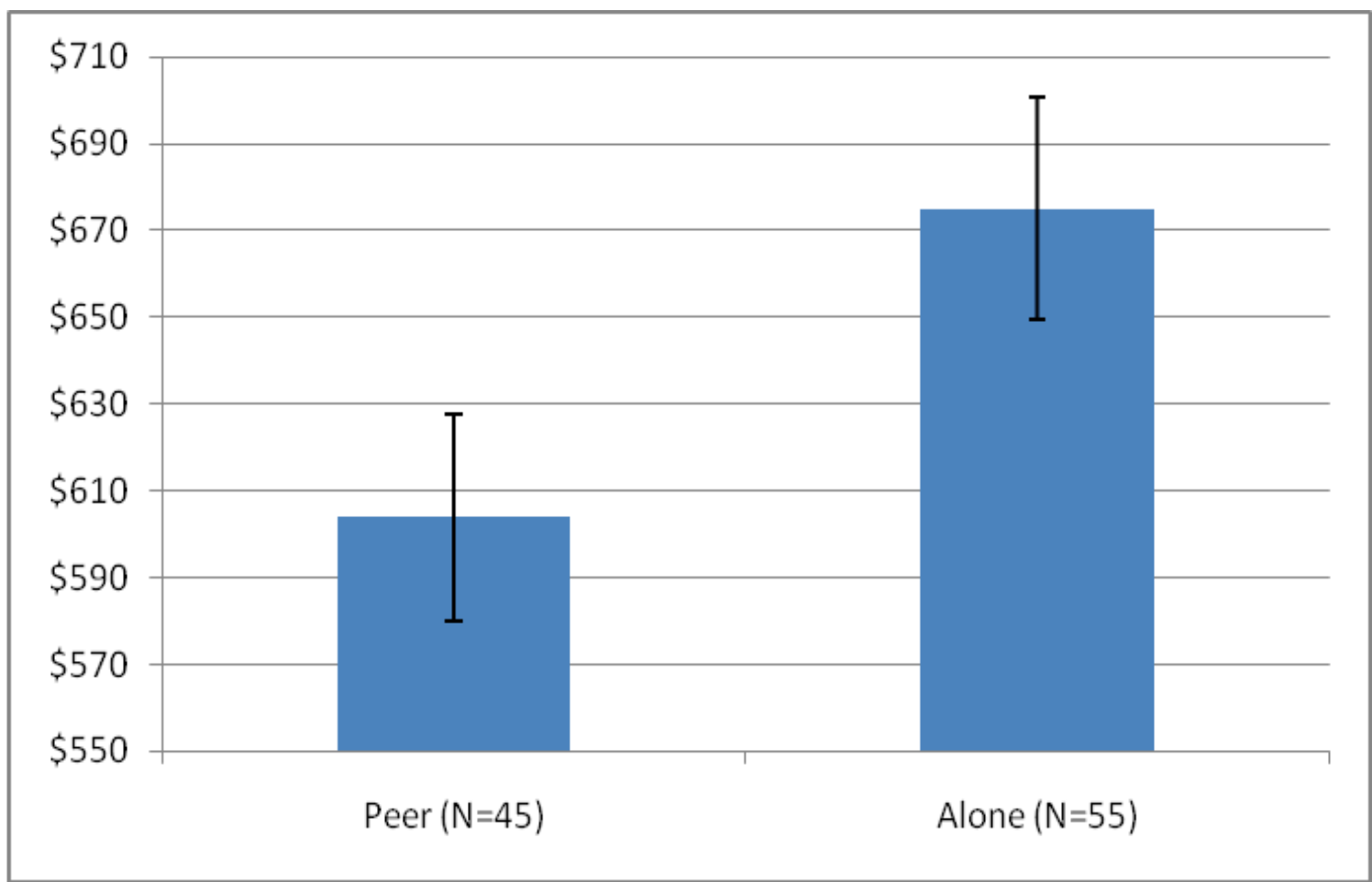

Note: Error bars represent one standard error above and below the mean. 
Figure 3. Discount Functions of 18- to 20-Year-Olds Alone and With Peers.

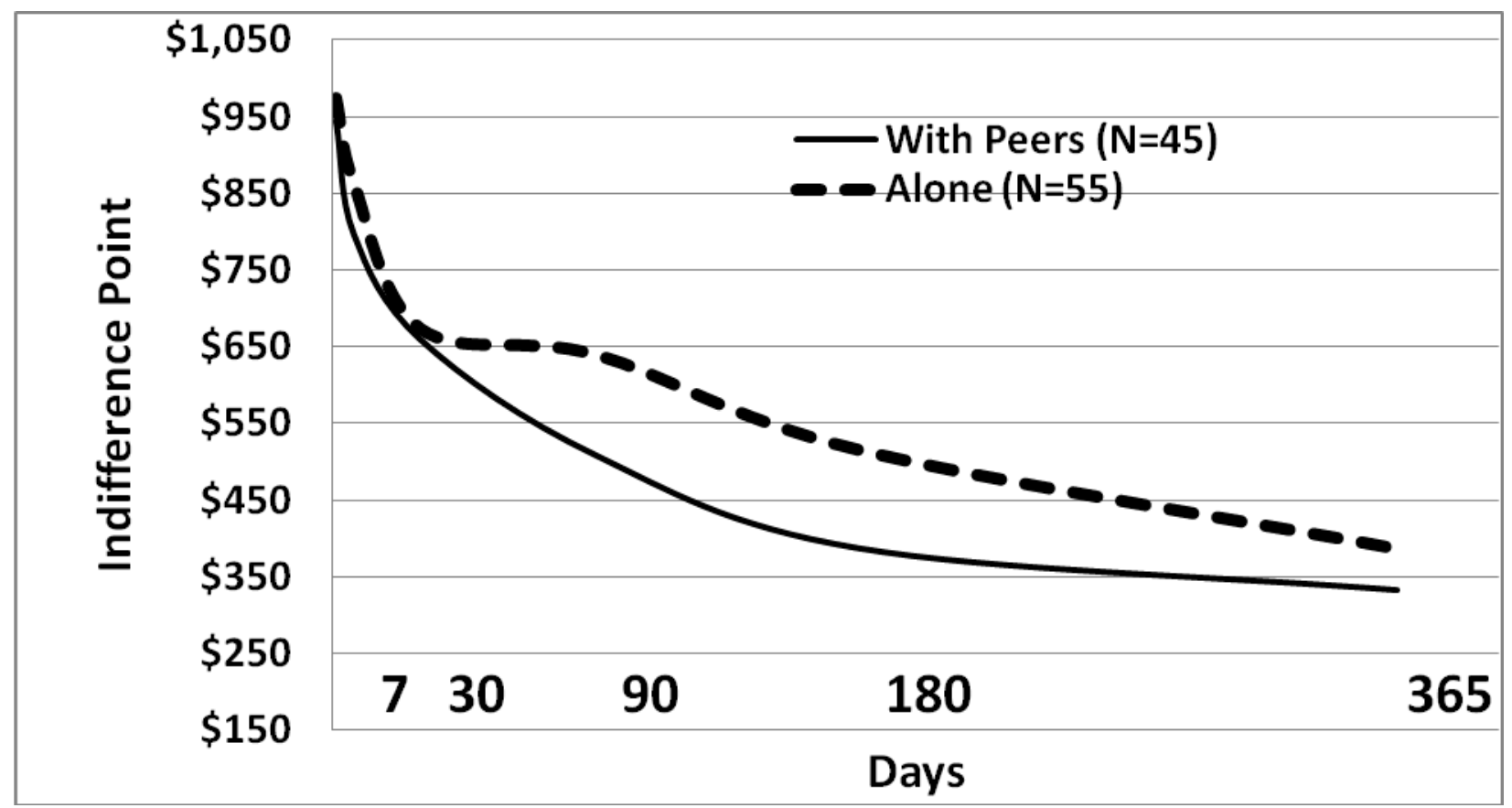


Figure 4. Average Indifference Points as a Function of Age and Peer Presence.

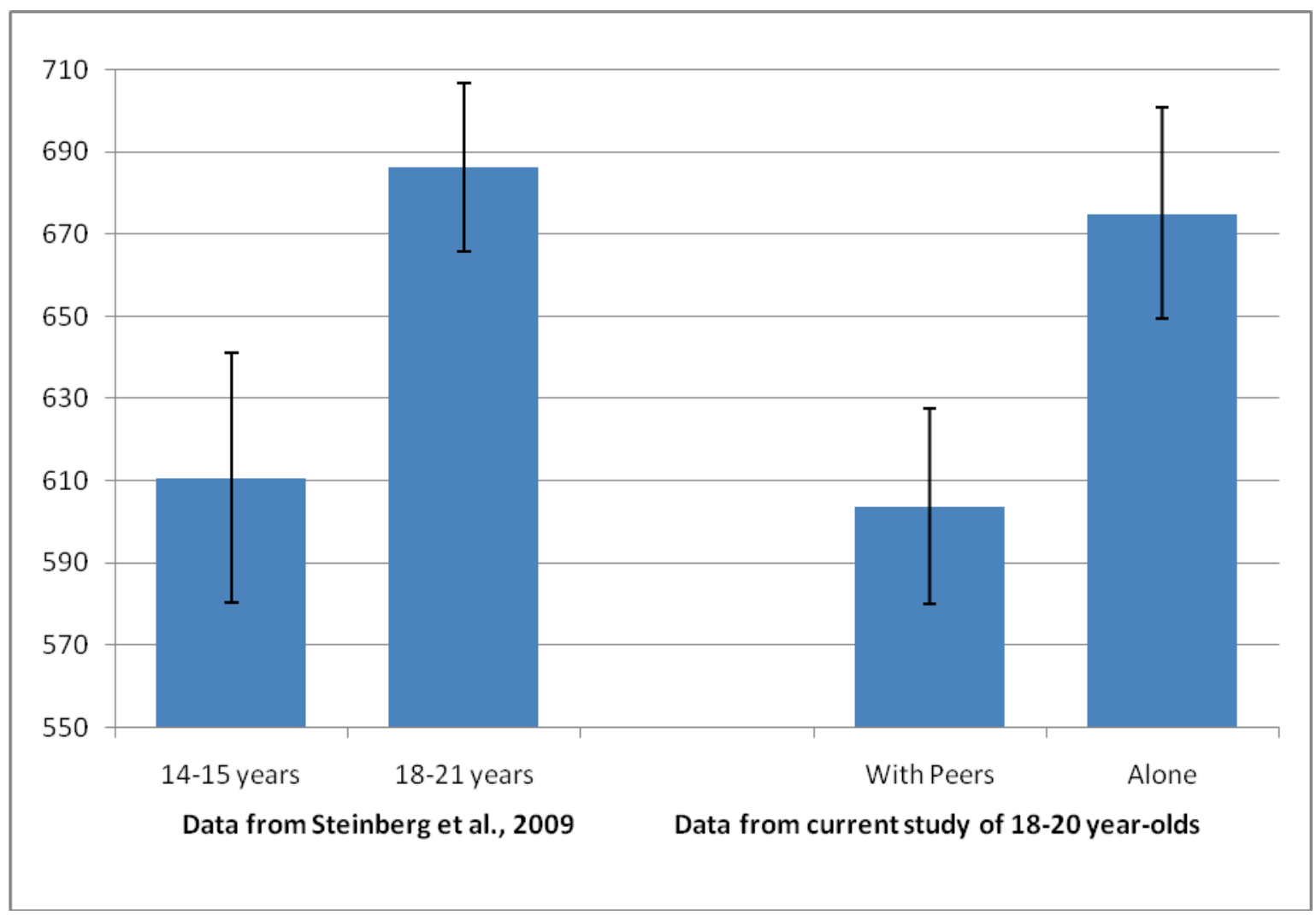

